\title{
Microwave/Millimeter Wave Metamaterial Development Using the Design of Experiments Technique
}

\author{
Daniela Staiculescu, Nathan Bushyager, Manos Tentzeris \\ Georgia Institute of Technology \\ Atlanta, GA, 30332, U.S.A. \\ daniela@ece.gatech.edu
}

\begin{abstract}
The successful use of Design of Experiments (DOE) approach in a feasibility and design study of a metamaterial structure is presented. The frequency of interest is $40 \mathrm{GHz}$ and the technology used is multilayer Low Temperature Cofired Ceramic (LTCC). The chosen approach for the Double Negative Metamaterial implementation is a loaded Coplanar Waveguide (CPW) transmission line. The design goals are a resonant frequency of $40 \mathrm{GHz}$ and minimum insertion loss at that frequency. The electromagnetic performance of the loaded transmission line is simulated with a full wave time domain commercial simulator. The results of these simulations are incorporated into the DOE technique. First, the significant factors in achieving each of the goals are identified, then statistical models are developed for the two output variables and applied to optimize the structure.
\end{abstract}

Keywords: optimization, metamaterials, statistical, design of experiments

\section{Introduction}

The concept of metamaterials, or left-handed materials (LHM), double negative (DNG), negative refractive index (NRI) materials, has been around since the late 1960's, but only recently has it gained a lot of interest since practical implementation solutions emerged. These materials exhibit phase and group velocities of opposite signs and a negative refractive index in certain frequency ranges, both characteristics making them desirable for RF and microwave applications. One of the implementation approaches starts from the equivalent transmission line model and artificially loads a host line with a dual periodical structure of series capacitors and shunt inductors. The length of the period and the value of the capacitors and inductors determine the frequency band in which the material has DNG behavior. One of the main challenges for the high frequency implementation, where the dimension become smaller and the process design rules become very restrictive, is the choice of the inductor and capacitor geometry to obtain the required left-handed passband and minimum insertion loss at the desired operating frequency. The goal of this paper is to design and optimize, for the first time, a metamaterial structure for $40 \mathrm{GHz}$. The current design methods do not take into account the specific effect of each of the factors involved in the design process and the degree these factors interact with each other. The use of DOE (Design of Experiments) allows these goals to be achieved.

\section{Metamaterials background}

Metamaterials are artificially loaded media which employ negative permittivity and permeability in finite frequency ranges [1]. One of the implementations of such structures are arrays of wires and 
split-ring resonators [2], [3]. These are complicated 3D systems that are difficult to apply for RF and microwave circuits. A more practical implementation uses transmission lines periodically loaded with lumped element networks [4], [5]. The starting point is the transmission line model presented in Fig. 1 (a).

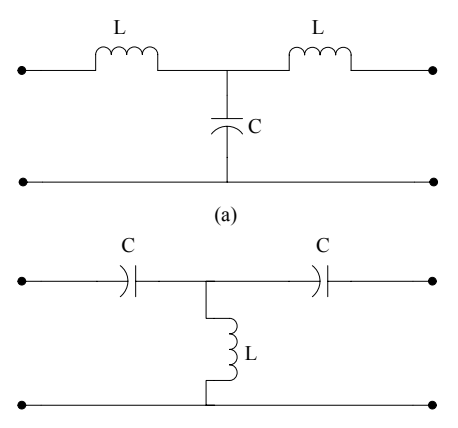

(b)

Fig. 1. Transmission line model (a) conventional (b) dual.

The equivalence between the distributed $\mathrm{L}$ and $\mathrm{C}$ for the transmission line and the permittivity and permeability of the medium is expressed as $\varepsilon=C, \mu=L$. By periodically loading this transmission line with its dual in Fig. 1 (b), the values of $\varepsilon$ and $\mu$ change as follows [4]:

$$
\varepsilon_{\text {eff }}=\varepsilon-\frac{1}{\omega^{2} L d}, \quad \quad \mu_{\text {eff }}=\mu-\frac{1}{\omega^{2} C d}
$$

where $\varepsilon$ and $\mu$ are the distributed inductance and capacitance of the host transmission line. It is obvious from (2) that for certain values of $L, C$ and $d$, the effective permittivity and permeability of the medium becomes negative for some frequency ranges. In these ranges, the refractive index is negative, and the phase and group velocities have opposite signs.

\section{DOE background}

A design of experiments is a series of tests in which a set of input variables or factors is purposely changed so that the experimenter can observe and identify the reasons for changes in the output response. Previous work shows the use of design of experiments in modeling of $\mathrm{RF} / \mathrm{microwave}$ circuits [6]. The factorial designs are used in experiments involving several factors where the goal is the study of the joint effects of the factors on a response. Prior knowledge of the analyzed system is required for choosing the factors and their studied ranges. The $2^{k}$ factorial design is the simplest one, with $k$ factors at 2 levels each. It provides the smallest number of runs for studying $k$ factors and is widely used in factor screening experiments [7].

This paper shows the first use of the DOE in a study of metamaterial design for frequencies as high as $40 \mathrm{GHz}$, saving a lot of time and frustration compared to the traditional trial-and-error approach.

\section{Metamaterial structure analysis and design}

The proposed structure is implemented in Low Temperature Cofired Ceramic (LTCC) technology, and the host transmission line is a $75 \Omega$ coplanar waveguide $(\mathrm{CPW})$. The advantage of the $\mathrm{CPW}$ is the ease to build shunt lumped elements due to the availability of the ground plane on the same layer as the signal, eliminating the need for vias. The series capacitors and shunt inductors are implemented as shown in Fig. 2. 


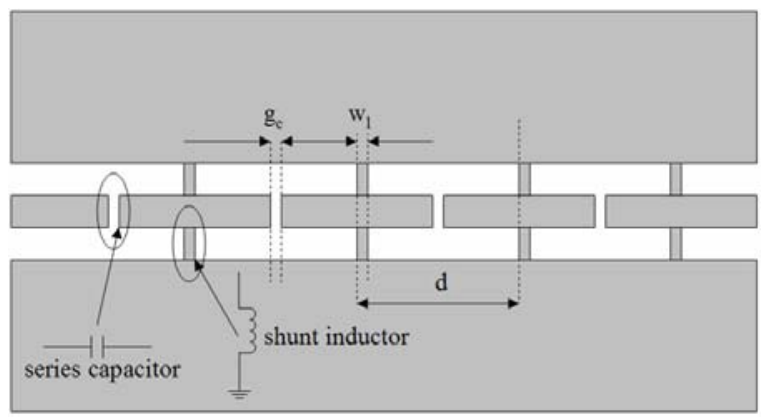

Fig. 2. CPW implementation of metamaterial structure.

The preliminary analysis of the structure dispersion diagram showed that the factors that influence the system performance the most are the values of the series capacitors and shunt inductors, as well as the length of the period. The experiment has been set for a constant characteristic impedance of $75 \Omega$, so the width of the signal line and the gap for the CPW are kept constant. Therefore, the three variables in the experiment are the gap of the capacitor $g_{c}$, the width of the inductive line $w_{l}$, and the length of the period $d$. A full factorial experiment with three factors consists of $2^{3}=8$ treatment combinations. The two levels chosen for each input variable have been controlled by the fabrication process and are presented in Table I.

\begin{tabular}{|c|c|c|c|}
\hline Variable & $g_{c}(\boldsymbol{\mu m})$ & $w_{l}(\boldsymbol{\mu m})$ & $\boldsymbol{d}(\boldsymbol{\mu m})$ \\
\hline "G-"level & 100 & 75 & 1600 \\
\hline${ }^{\text {"6+"6 level }}$ & 200 & 125 & 2000 \\
\hline
\end{tabular}

Table I. Variables for the $2^{3}$ experiment

The output variables are the resonant frequency in the first LH passband $f_{\text {res }}$ and the value of the insertion loss at that frequency $I L$. The eight simulations have been run in MicroStripes TLM Modeler and the results are presented in Table II.

\begin{tabular}{|c|c|c|c|c|c|}
\hline Run & $\boldsymbol{g}_{\boldsymbol{c}}$ & $\boldsymbol{w}_{\boldsymbol{l}}$ & $\boldsymbol{d}$ & $\begin{array}{c}\boldsymbol{f}_{\text {res }} \\
(\mathbf{G H z})\end{array}$ & $\begin{array}{c}\boldsymbol{I L} \\
(\mathbf{d B})\end{array}$ \\
\hline $\mathbf{1}$ & - & - & - & 48.1 & 9.2 \\
\hline $\mathbf{2}$ & + & - & - & 38.7 & 5 \\
\hline $\mathbf{3}$ & - & + & - & 47.7 & 10 \\
\hline $\mathbf{4}$ & + & + & - & 39.3 & 7 \\
\hline $\mathbf{5}$ & - & - & + & 48.3 & 12 \\
\hline $\mathbf{6}$ & + & - & + & 39 & 7 \\
\hline $\mathbf{7}$ & - & + & + & 48.4 & 13.5 \\
\hline $\mathbf{8}$ & + & + & + & 40.4 & 9 \\
\hline
\end{tabular}

Table II. $2^{3}$ experiment

Analysis of Variance (ANOVA) statistical analysis [7] has been performed using a user-friendly specialized commercial software. ANOVA reveals the statistical significance of all the input variables and of their interactions and generates regression models of the outputs as a function of the inputs. The first important result we obtained for this case is that the width of the inductor strip is not statistically significant for any of the output variables and therefore can be eliminated from further analysis. The 
capacitor gap is only significant for the insertion loss, and it will be included in the regression models. The length of the period is significant for both the outputs. The regression models are given by:

$$
\begin{aligned}
& f_{\text {res }}=82.26+0.0056 \cdot g_{c}(\mu \mathrm{m})-0.0219 \cdot d(\mu \mathrm{m}) \\
& I L=23.51+0.0256 \cdot g_{c}(\mu \mathrm{m})-0.0104 \cdot d(\mu \mathrm{m})
\end{aligned}
$$

These results are valid only for the intervals in Table I. It can be observed that the design process has been reduced dramatically, first by eliminating a variable from the analysis, then by obtaining explicit equations for the outputs as a function of the inputs. The structure is also optimized with the following goals $f_{\text {res }}=40 \mathrm{GHz}, I L=\min$.

The optimization is performed using the statistical analysis software and the results are $f_{\text {res }}=$ $40 G H z$ and $I L=5.72 d B$ These are obtained for $g_{c}=100 \mu m$ and $d=1953.4 \mu m$. The high insertion loss is due to the difficulty to implement the distributed lumped elements at the high frequencies with the limitations given by the design rules of the fabrication process. The next steps include the optimization of the lumped elements, the extension of the statistical analysis to more sophisticated tools and the validation of the optimized structure with test structure fabrication and measurements.

\section{Conclusions}

This paper presents a method in which deterministic electromagnetic simulation tools and statistical modeling methods can be used to optimize RF and microwave metamaterials. To prove the concept, a benchmarking geometry of a CPW transmission line loaded with series capacitors and shunt inductors was chosen. The two optimized factors were the resonating frequency and the insertion loss. Three physical parameters of the structure were chosen as optimization variables. The results of the hybrid electromagnetic-statistical analysis generated statistical models that could be used to predict the metamaterial performance based on the geometry of the structure. These models could then be used to optimize the structure with respect to desired performance, enabling the system-level optimization of the geometry in quick and inexpensive way. In this case, the center frequency was chosen and the insertion loss was minimized to exemplify the possibilities of the method.

The proposed approach can be easily extended to a larger number of design variables and optimized figures of merit. The main challenge in this case is the implementation of the distributed lumped elements. In this way, the behavior and challenges of a complex meatamaterial system could be predicted at the beginning of the design process, leading to significant time savings and a much shorter design cycle of added functions, while achieving the design and optimization goals in a simple and elegant manner.

\section{References}

[1] D. R. Smith, W. J. Padilla, D. C. Vier, S. C. Nemat-Nasser, S. Schultz, "Composite Medium with Simultaneously Negative Permeability and Permittivity", Phys. Rev. Lett., vol. 84, no. 18, pp. 4184-4187, May 2000.

[2] E. Ozbay, K. Aydin, E. Cubukcu, M. Bayindir, "Transmission and Reflection Properties of Composite Double Negative Metamaterials in Free Space", IEEE Trans. Antennas and Propagation, vol. 51, no. 10, pp. 2592-2595, October 2003.

[3] R. A. Shelby, D. R. Smith, S. Schultz, "Experimental verification of a negative index of refraction", Science, vol. 292, pp. 77-79, April 2001.

[4] G. V. Eleftheriades, A. K. Iyer, P. C. Kremer, "Planar Negative Refractive Index Media Using Periodically $L-C$ Loaded Transmission Lines", IEEE Trans. Microwave Theory \& Tech., vol. 50, no. 12, pp. 2702-2712, December 2002.

[5] A. Grbic, G. V. Eleftheriades, "Experimental Verification of Backward-Wave Radiation from a Negative Refractive Index Material", Journal of App. Physics, vol. 92, no. 10, pp. 5930-5935, November 2002.

[6] D.Staiculescu, J.Laskar, E.M.Tentzeris, "Design Rule Development for Microwave Flip-Chip Applications", IEEE Trans. Microwave Theory \& Tech., Vol.48, No.9, pp.1476-1481, September 2000.

[7] D.C. Montgomery, Design and Analysis of Experiments, New York: J. Wiley \& Sons, 1997. 